\title{
Quantitative pigment extraction analysis for human pluripotent stem cell derived retinal pigment epithelial cells
}

\author{
Rieck, Jochen ${ }^{1}$, Juuti-Uusitalo, Kati ${ }^{1}$, Autio, Reija ${ }^{2}$, Hytönen, Vesa ${ }^{1}$, Skottman, Heli $^{1}$ \\ ${ }^{1}$ Faculty of Medicine and Life Sciences, BioMediTech Institute, University of Tampere, Finland \\ ${ }^{2}$ Faculty of Social Sciences, University of Tampere, Finland
}

\begin{abstract}
Pigmentation is a typical feature for retinal pigment epithelial cells (RPE), and each RPE cell represents individual characteristics. Although, type and intensity of pigmentation may vary, still most cells are pigmented to some extent.

In this study, we set-up a standardized quantitative melanin content analysis for human embryonic stem cell (hESC) derived RPEs (hESC-RPE) and compared this method to an image based pigment quantification technique. The optical quantification of pigmentation was done from micrographs of hESC-RPE. The extracted pigment was quantitated with spectral analysis, with UV-Vis spectroscopy, and the size-distribution with dynamic light scattering.

The results revealed that the measured particle sizes of extracted melanin corresponded to known sizes extracted melanosomes. In addition, the optical pigmentation and absolute melanin concentration were clearly correlating.

Our data suggests that image analysis and quantitative melanin content analysis can be interchangeably utilized in pigmentation quantitation: if the relative pigmentation of hESC-RPE cells is desired to be estimated with a non-invasive method then image analysis is the choice of method, but if the exact amount of melanin needs to be evaluated, then the new melanin extraction method presented here, should be chosen.
\end{abstract}

Keywords-hESC-RPE, Melanin, Pigmentation quantification

\section{INTRODUCTION}

The retinal pigment epithelium (RPE) is a highly melanized cell monolayer, situated between neural retina and choroid in the back of the eye. The RPE fulfills essential tasks for the neighboring neural retina providing the long-term preservation of retinal integrity and visual functions by absorbing stray light, recycling of retinoid, supplying nutrients, secreting growth factors and phagocytosing of shed photoreceptor outer segments $(1,2)$. The RPE cells differentiated from human embryonic stem cells (hESCs) and human induced pluripotent stem cells (hiPSCs) are proposed to be suitable cell source for transplantation therapy in retinal diseases (3). The current differentiation protocols for the production of RPE cells mainly rely on spontaneous (4), or more directed differentiation methods (5). The characteristics of these generated RPEs should be thoroughly analyzed for down-stream applications.
The mature RPE exhibits three major types of pigmentation: melanin containing melanosomes, lipofuscin and melanolipofuscin. While melanosomes are formed during embryogenesis the accumulation of lipofuscin and melanolipofuscin are a symptom of aging. In cultured hESC-RPE, pigment density initially decreases in the course of cell divisions, which results in a gradual dilution of pigment among the daughter cells, but as the cells become confluent de novo synthesis of pigment can be observed $(6,7)$. By day 30 , the pigment density is comparable to that in native human RPE (6). Among other features, hESC-RPE are expected to exhibit the same type of pigmentation as native RPE cells.

The pigmentation density at a distinct time point can serve both as a marker for the efficiency of a differentiation protocol and as a guideline for the stage of maturation. Therefore, in this study we introduce a protocol for a standardized quantitative melanin content analysis for RPE cells, comparing this method to an image based pigment quantification technique in respect to sensitivity and reproducibility.

\section{MATERIALS AND METHODS}

Each set of cultured hESC-RPE cells $(A)$ was processed as follows: in the end of culture (42d or $135 \mathrm{~d}$ ) cells were photographed $(B)$, and the pigment was chemically extracted $(C)$. Thereafter the pigment concentration $(C)$, spectral properties $(D)$ and particle size composition $(D)$ of the extracts were determined.

\section{A. hESC and RPE differentiation}

Two hESC lines derived at our laboratory were used in this study: Regea08/017 (46,XX) and Regea08/023 (46,XY) (8). The hESCs were differentiated to RPE as floating cell aggregates as described previously $(4,9)$. For the expansion of RPE cells, pigmented areas were manually isolated, dissociated and replated as previously described $(4,9)$. For the experiments the cells were seeded on Collagen IV -coated 24 well plates (Sigma-Aldrich, St. Louis, USA) and cultured for $42 \mathrm{~d}$ until light pigmentation was visible; or up to $136 \mathrm{~d}$. 


\section{B. Optical pigmentation quantification}

The optical pigmentation analysis from bright field micrographs was done as in (9), from five randomly selected areas.

\section{Chemical melanin extraction}

Cells were dissociated with TrypLETM Select (Life Technologies, Carlsbad, USA) and counted. The cells were washed twice with $0.5 \mathrm{ml}$ PBS, pelleted at $16.000 \mathrm{~g}, 5 \mathrm{~min}$ at $4{ }^{\circ} \mathrm{C}$. The floating cell-pellets were sonicated in an ice-cooled Bioruptor Standard device (Diagenode, Seraing, Belgium) with the highest intensity, 7 minutes, $30 \mathrm{sec}$ intervals. Afterwards, the cell debris was pelleted, $16.000 \mathrm{~g}, 5 \mathrm{~min}$ at $4 \mathrm{C}$, and pellet was washed with $500 \mu \mathrm{l} \mathrm{cool} \mathrm{wash-solution}(0,25$ $\%$ Triton X-100, $2 \%$ SDS in PBS) and re-pelleted (16.000 g, 5 minutes at $8{ }^{\circ} \mathrm{C}$ ), the supernatant discarded and replaced with $500 \mu \mathrm{l}$ cool PBS. This centrifugation step was repeated and the supernatant was then replaced with $50 \mu \mathrm{l} 1 \mathrm{~N} \mathrm{NaOH}$ (supplemented with $10 \%$ DMSO), followed by an incubation-step at $80^{\circ} \mathrm{C}$; until the pellet was completely dissociated i.e. the initially clear $\mathrm{NaOH}$ had become brown. Finally, the melanin contents of all extracts were determined with an EnVision 2104 Multilabel reader (Perkin Elmer, Waltham, USA) by measuring the absorbance between 330 and $340 \mathrm{~nm}$ (theoretical absorbance maximum of melanin, (10)), using a low auto-fluorescence DELFIA 8x12 strip micro plate (Perkin Elmer, Waltham, USA). Synthetic melanin [100 $\mu \mathrm{g} / \mathrm{ml}]$ (Sigma-Aldrich, St. Louis, USA) served as a standard.

D. Spectral analysis and size distribution analysis with $d y$ namic light scattering

The extracted pigment was identified with UV-Visspectra (290-890 nm) of both hESC-RPE extracts and synthetic melanin [100 $\mu \mathrm{g} / \mathrm{ml}]$ (Sigma-Aldrich, St. Louis, USA) using a LAMBDA $35 \mathrm{UV} / \mathrm{V}$ is spectrometer (Perkin Elmer, Waltham, USA) with a $50 \mu \mathrm{l}$ quartz-cuvette. Finally the pigment extracts from heavily pigmented and lightly pigmented hESC-RPE samples and synthetic melanin were analyzed with a Zetasizer Nano device (Malvern Instruments, Malvern, UK), using a $50 \mu 1$ low fluorescence cuvette (Analysissettings: Dispersant RI: 1.330 , Viscosity: $1.5678 \mathrm{mPa} \cdot \mathrm{s})$.

\section{E. Statistical analysis}

The statistical analysis was performed using SPSS analyzing software (IBM, Armonk, USA), and statistical significance was determined with two tailed Mann-Whitney U test.

\section{RESULTS}

\section{A. Optical pigmentation quantification}

The image analysis of bright field micrographs (Figure 1) of heavily and lightly pigmented hESC-RPE revealed that after $42 \mathrm{~d}$ the optical pigmentation values ranged from 4.89 to 7.15. After $136 \mathrm{~d}$ of culture with heavy pigmentation, the optical pigmentation ranged from 1.49 to 1.62 .

\section{B. Chemical melanin extraction quantification}

The photometric concentration determination from the same samples resulted in absolute pigment concentrations; ranging from 89 to $390 \mu \mathrm{g} / \mathrm{ml}$. Subsequently, these values were normalized by dividing the measured pigment concentrations by the cell count (Figure 1).

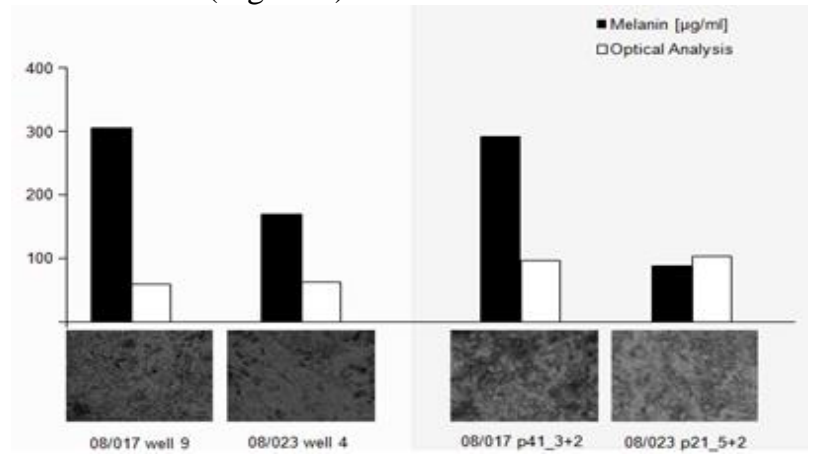

Figure 1: Representative images of examined lightly (left) and heavily pigmented hESC-RPE with corresponding melanin and relative (optical) pigmentation values (without background subtraction).

\section{Analysis of pigmentation}

The visual comparison of the pigmentation from cells with the same culture time and similar cell count, the Regea08/017 hESC-RPEs displayed a denser and darker pigmentation than Regea08/023 hESC-RPEs. The normalized melanin values of the light-pigmented cells differed significantly from those, obtained from the heavily pigmented ones $(p=0.03)$. There was a significant difference between melanin values of lightly pigmented samples from different cell lines: The Regea08/017 hESC-RPEs higher than Regea08/023 hESCRPEs ( $\mathrm{p}<0.001)$. The lightly pigmented (Regea08/017) samples $(n=12)$ yielded optical pigmentation values, ranging from 5.8 to 6.3 (median 6.08) and melanin concentrations, ranging from 266 to $390 \mu \mathrm{g} / \mathrm{ml}$ (median $335 \mu \mathrm{g} / \mathrm{ml}$. The lightly pigmented Regea08/023 hESC-RPEs $(n=12)$, yielded optical pigmentation values, ranging from 4.7 to 5.9 (median 5.3) and melanin concentrations, ranging from 145 to 209 $\mu \mathrm{g} / \mathrm{ml}$ (median $179 \mu \mathrm{g} / \mathrm{ml}$ ). The heavily pigmented samples 
(Regea08/017 and Regea08/023, n=4) had optical pigmentation values ranging from 1.49189 to 1.62916 (median 1.56600 ), and melanin values, ranging from 89 to $291 \mu \mathrm{g} / \mathrm{ml}$ (median $174 \mu \mathrm{g} / \mathrm{ml}$ ) (Figure 2).

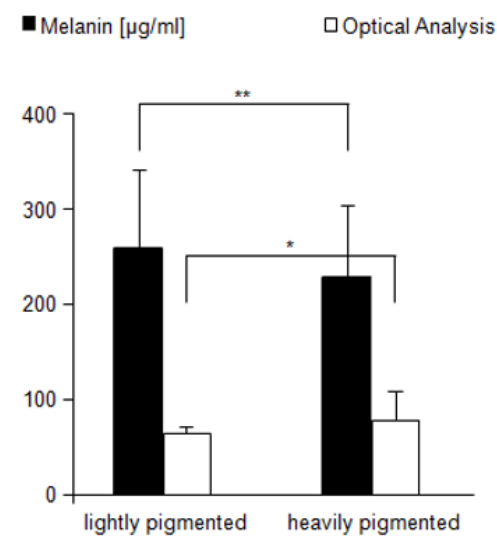

Figure 2: Comparison of absolute melanin-values and relative optical pigmentation-values (without background-subtraction). The heavily pigmented samples $n=4$, lightly pigmented samples $n=12$ form both (Regea08/017 and $2 x$ Regea08/023) cell lines $\left(* p \leq 0.05,{ }^{* *} p \leq 0.01\right)$.

\section{Comparison of the methods}

The statistical correlation analysis revealed that optical pigmentation values and melanin values are clearly correlating. When all samples were analysed together, the correlation between the optical values and melanin concentration values was $0.63(\mathrm{p}<0.001)$. The correlation was even higher when analysing only lightly pigmented samples $(r=0.76, \mathrm{p}<0.001)$, and further the correlation of the Regea08/017 samples was more obvious, compared to the Regea08/023 samples. In contrast, a correlation of the values resulting from the heavily pigmented samples could not be confirmed possibly due to small sample number and variation between the heavily pigmented Regea08/017 and Regea08/023 samples. The pigmentation values of the lightly pigmented samples differed statistically significantly from those of the heavily pigmented samples.

\section{E. Spectral analysis}

The spectral analysis of synthetic melanin and two randomly selected extracts from both cell lines resulted in a complex spectra (Figure 3.), but there was a high similarity between the spectrum from synthetic melanin and the spectra from hESC-RPE extracts (Figure 3).
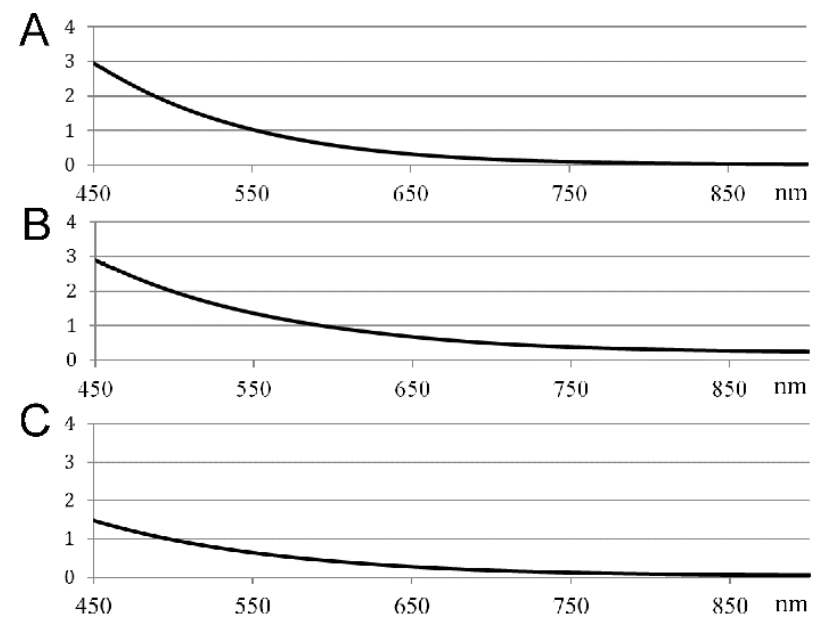

Figure 3: Spectral analysis (450-900nm) of synthetic melanin (A), Regea08/017 (B) and Regea08/023 (C) hESC-RPEs.

\section{F. Dynamic light scattering analysis}

The size distribution analysis revealed a particle size between 100 and $800 \mathrm{~nm}$ for synthetic melanin (Figure 4A). The diameters of particles detected in the pigment extracts varied from 80 to $8000 \mathrm{~nm}$ in the case of Regea08/017 cells (Figure 4B) and from 800 to $8000 \mathrm{~nm}$ in extracts of Regea08/023 cells (Figure 4C).

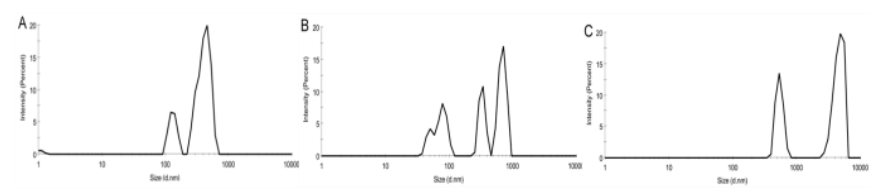

Figure 4: Particle size-distribution recorded from synthetic melanin (A), Regea08/017 (B) and Regea08/023 (C) hESC-RPEs

\section{DISCUSSION}

In this study, we aimed to develop new melanin extraction method for hESC-RPE cells compared to the previous one $(11,12)$. The UV-Vis-spectra recorded here from synthetic melanin and from hESC-RPE extracts illustrates the complexity and the polymeric nature of the melanin-extracts. This result is in accordance with the published literature (10, 13, 14). Melanosomes of the RPE are non-transparent and appear in two shapes: elliptical melanosomes $(0.5$ to $1.0 \mu \mathrm{m}$ in width, 1.0 to $2.5 \mu \mathrm{m}$ in diameter), spherical or oval melanosomes (smaller one 0.5 to $1.0 \mu \mathrm{m}$, larger ones 1.5 to $2.0 \mu \mathrm{m})(15,16)$. In Sepia, eumelanin sizes range from 3 to $286 \mu \mathrm{m}$ in the long dimension and other more densely packed particles, that vary in their lateral dimension from 45 to 230 $\mathrm{nm}(17,18)$. This, relatively wide size distribution, was also 
documented in this study. In addition the high variation in size $(80-8000 \mathrm{~nm})$, detected in here can be explained by the tendency of melanin to form aggregates.

The other aim was to evaluate if the pigment-extraction protocol could verify pigmentation values gained with the optical pigmentation analysis. The pigmentation analysis from the hESC-RPEs has previously been done with image analysis from the bright field micrographs $(9,19,20)$ or by quantitating the extracted melanin (3) but comparison has not been previously done. Here our results demonstrated that optical pigmentation values and melanin values were clearly correlating. Our conclusion is that the pigmentation values obtained with image analysis method can be used to evaluate non-invasively the relative amount of pigmentation in hESCRPEs. However, in order to acquire absolute melanin concentration then the invasive, "end-point analysis", of quantitative pigment extraction should be used.

\section{ACKNOWLEDGEMENT}

This work was supported by the Finnish Funding Agency for Technology and Innovation, Health Research Council of the Academy of Finland (grant numbers 218050 and 137801). The funders had no role in study design, data collection and analysis, decision to publish, or preparation of the manuscript. Outi Melin, Outi Heikkilä, Hanna Pekkanen and Jenna Tainio are thanked for excellent technical assistance with cells.

\section{Conflict Of InTERest}

The authors declare that they have no conflict of interest.

\section{REFERENCES}

1. Fuhrmann, S., Zou, C., Levine, E. M. Retinal pigment epithelium development, plasticity, and tissuehomeostasis. Experimental Eye Research 2013:1-10.

2. Juuti-Uusitalo, K., Vaajasaari, H., Ryhänen, T., Narkilahti, S., Suuronen, R., Mannermaa, E., Kaarniranta, K., Skottman, H. Efflux Protein Expression in Human Stem Cell-Derived Retinal Pigment Epithelial Cells. PLoS ONE 2012;7:1-10.

3. Schwartz, S. D., Hubschman, J.-P., Heilwell, G. et al. Embryonic stem cell trials for macular degeneration:a preliminary report. Lancet 2012;379:713-20.

4. Vaajasaari, H, Ilmarinen, T, Juuti-Uusitalo, K, Rajala, K, Onnela, N, Narkilahti, S, Suuronen, R, Hyttinen, J, Uusitalo, H, Skottman, H. Toward the defined and xeno-free differentiation of functionalhuman pluripotent stem cell-derived retinal pigment epithelialcells. Molecular Vision 2011;17:558-75.
5. Hsiung, J., Zhu, D., Rodriguez, A., Hinton, D., R. Retinal Pigment Epithelial Cell Conditioned Medium Enhances the Yield ofRPE Cells Differentiated from Human Embryonic Stem Cells. Clinical \& Experimental Pathology 2014;5.

6. Boulton ME. Studying melanin and lipofuscin in RPE cell culture models. Experimental Eye Research 2014;126:61-7.

7. Flood, M. T., Gouras, P., Kjeldbye, H. Growth characteristics and ultrastructure of human retinal pigment epithelium in vitro. Invest. Ophthalmol. Vis. Sci. 1980;19:1309-20.

8. Skottman H. Derivation and characterization of three new humanembryonic stem cell lines in Finland. In Vitro Cellular \& Developmental Biology - Animal In Vitro Cellular \& Developmental Biology 2010;46:206-9.

9. Sorkio, A., Hongisto, H., Kaarniranta, K. et al. Structure and Barrier Properties of Human EmbryonicStem Cell-Derived Retinal Pigment Epithelial CellsAre Affected by Extracellular Matrix Protein Coating. TISSUE ENGINEERING 2014;20:1-13.

10. Riesz, Jennifer. The spectroscopic properties ofmelanin. A thesis submitted for the degree of Doctor of Philosophy to the University of Queensland 2007.

11. d'Ischia, M., Wakamatsu, K., Napolitano, A., Ito, S. et al. Melanins and melanogenesis: methods, standards, protocols. Pigment Cell Melanoma Res. 2013;26:616-33.

12. Liu Y, Simon JD. Isolation and Biophysical Studies of Natural Eumelanins: Applications of Imaging Technologies and Ultrafast Spectroscopy. Pigment Cell Research 2003;16:606-18.

13. Meredith, P., Sarna, T. The physical and chemical properties of eumelanin. Pigment Cell Res. 2006;19:572-94.

14. Peles, D. N., Simon, J. D. The UV-Absorption Spectrum of Human Iridal Melanosomes:A New Perspective on the Relative Absorption of Eumelanin and Pheomelanin and its Consequences. Photochemistry and Photobiology 2012;88:1378-84.

15. Schraermeyer, U., Heimann, K. Current Understanding on the Role of Retinal Pigment Epithelium and its Pigmentation. Pigment Cell Research 1999;12:219-36.

16. Sarna, T., Burke, J. M., Korytowski, W., Rozanowska, M., Skumatz, C., M., B., Zareba, M. Loss of melanin from human RPE with aging: possible roleof melanin photooxidation. Experimental Eye Research 2003;76:8998.

17. Vitkin IA, Woolsey J, Wilson BC, et al. OPTICAL AND THERMAL CHARACTERIZATION OF NATURAL (Sepia officinalis) MELANIN. Photochem Photobiol 1994;59:455-62.

18. Zeise L, Chedekel MR. Melanin Standard Method: Particle Description. Pigment Cell Research 1992;5:132-42.

19. Nanni, L., Paci, M., Caetano dos Santos, F. L., Skottman, H. et al. Texture Descriptors Ensembles Enable Image-Based Classification of Maturation of Human Stem Cell-Derived Retinal Pigmented Epithelium. PLoS ONE 2016.

20. Kamao, H., Mandai, M., Wakamiya, S. et al. Objective Evaluation of the Degree of Pigmentation in Human Induced Pluripotent Stem Cell-Derived RPE. IOVS 2014;55:8309-18.

Enter the information of the corresponding author:

Author: Heli Skottman

Institute: Faculty of Medicine and Life Sciences, BioMediTech Institute, University of Tampere

Street: $\quad$ Lääkärinkatu 1, ARVO building

City: Tampere

Country: Finland

Email: $\quad$ heli.skottman@uta.fi 